\title{
Des « livres sacrés ». Fiction et idée de la bibliothèque au Moyen Âge
}

\section{Dietmar Rieger}

\section{(2) OpenEdition \\ 1 Journals}

Édition électronique

URL : https://journals.openedition.org/crm/902

DOI : 10.4000/crm.902

ISSN : 1955-2424

Éditeur

Honoré Champion

Édition imprimée

Date de publication : 15 février 2000

ISSN : 1272-9752

Référence électronique

Dietmar Rieger, «Des « livres sacrés ». Fiction et idée de la bibliothèque au Moyen Âge », Cahiers de recherches médiévales [En ligne], 7 | 2000, mis en ligne le 03 janvier 2007, consulté le 15 décembre 2022. URL : http://journals.openedition.org/crm/902 ; DOI : https://doi.org/10.4000/crm.902

Ce document a été généré automatiquement le 15 décembre 2022.

Tous droits réservés 


\title{
Des « livres sacrés ». Fiction et idée de la bibliothèque au Moyen Âge
}

\author{
Dietmar Rieger
}

1 Avant d'essayer de mettre en lumière la bibliothèque du Moyen Âge imaginée en tant que fiction littéraire ou bien en tant qu'idée et idéal, il faut souligner que les changements et bouleversements qui se sont produits essentiellement depuis la fin du XII ${ }^{\mathrm{e}}$ siècle, ont certes mené à une différenciation fondamentale du savoir et des sciences, sans pour autant que cela ait eu des répercussions décisives sur l'idée, encore en quête d'harmonie, de la bibliothèque. Dans cette mesure, il est permis de dire qu'au Moyen Âge la bibliothèque telle qu'elle est imaginée est en retard par rapport à la réalité du savoir, voire même à la réalité de la bibliothèque.

2 La bibliothèque, au sens d'une collection de supports écrits (biblos) et d'un lieu où est conservée une telle collection (theke) - ces deux implications sémantiques sont très tôt concomitantes pour ce collage lexical gréco-latin ${ }^{1}$ - est au moins depuis le troisième millénaire avant J.-C. une composante importante de toute culture littéraire. Toutefois, aussi ancienne que soit la bibliothèque en tant que lieu de stockage du savoir écrit, en tant que forme institutionnalisée de la mémoire collective ${ }^{2}$, ce n'est qu'assez tardivement dans l'histoire des bibliothèques réelles qu'elle fait son apparition dans la littérature fictionnelle. Autrement dit : ce n'est qu'à partir d'un certain moment que la localisation du livre dans la bibliothèque produit sa propre inversion, c'est-à-dire la localisation de la bibliothèque dans le livre (de fiction). Même les premières "théories " bibliothéconomiques comme celle de Sénèque, qui, dans De tranquilitate animi, se fait l'avocat d'une bibliothèque pragmatique, pratique pour l'utilisateur, et fustige la démesure bibliophile, ou bien celle de Richard de Bury, lequel justifie au contraire la caritas librorum dans son Philobiblon, précèdent les bibliothèques véritablement fictionnelles. Avant la Renaissance, les protagonistes lisant ne sont pourtant pas rares dans les textes de fiction. La «pucelle» dans le Yvain de Chrétien de Troyes, laquelle lit à ses parents un «romanz» dans un «vergier » (v. 5360 et suiv.), une femme lisant son psautier dans le lai Yonec de Marie de France (v. 60), Francesca da Rimini et Paolo Malatesta, qui dans la Divina Commedia de Dante (Inferno, V, 127 et suiv.) lisent ensemble 
«Liancalotto»- et par cette lecture deviennent des amants tragiques -, ou encore Floire et Blancheflor dans le roman d'amour du même nom, qui lisent ensemble Ovide («Livres lisoient paienors / $\mathrm{u}$ ooient parler d'amors», vv. 231-232), ne sont que quelques exemples parmi bien d'autres. Certes, il y a les «galeries littéraires» de la poésie courtoise - dans un cadre de critique littéraire ou de satire jongleresque ${ }^{3}$, ou bien dans celui des performances poétiques lors des fêtes courtoises ${ }^{4}$ - et il y a aussi, entre autres, le fameux "catalogue» poétique de la bibliothèque de l'école de la cathédrale d'York établi par Alcuin. Cependant, on ne trouvera sans doute guère de véritable bibliothèque dans un contexte spécifiquement fictionnel.

Il faut néanmoins souligner le fait que, vers la fin du Moyen Âge, ces listes littéraires se multiplient et se concrétisent - passant de la mention de genres et de noms de poètes à celle d'œuvres précises. Cette évolution témoigne d'une importance croissante de la bibliothèque, non seulement en tant que telle, mais en particulier en tant que collection d'«individualités» livresques qui, dans un premier temps, continuent d'être maintenues ensemble par une espèce d'idée centraliste de la bibliothèque; mais elles sont pourtant en train de préparer leur future émancipation, laquelle apparaît aussi dans le fait, qu'en même temps, l'œuvre individuelle sort de plus en plus - et bientôt de manière généralisée, suite à l'invention de l'imprimerie - du collectif du manuscrit. $\mathrm{Ne}$ mentionnons que trois exemples de ces catalogues littéraires: une sorte de canon narratif vernaculaire est la longue liste d'œuvres désignées par des noms de héros, masculins ou féminins, appartenant en particulier à la matière de Bretagne et à la chanson de geste, qu'énumère un auteur anonyme (ou bien s'agit-il d'un certain Requis?) en prologue à son roman Richars li Biaus au cours de la première moitié du $\mathrm{XIV}^{\mathrm{e}}$ siècle, afin de présenter son propre roman en vers comme surpassant de loin toutes ces œuvres:

4 Tout chil que ie vous ai conté

N'ont de valour ne de bonté

Vaillant deus nois enviers chestui

Dont vous m'orrés conter ancui. (vv. 29-32)

Autre catalogue assez long, non fictionnel, mais unique au cours de la première moitié $\mathrm{du} \mathrm{XIV}^{\mathrm{e}}$ siècle à cause de ses références autobiographiques au rôle croissant de la langue vulgaire dans l'écrit, le catalogue de ses lectures favorites - avec parmi elles le Roman de la Rose - qu'établit Gilles li Muisis en 1350, dans le contexte de ses Méditations. Ce moine-poète aveugle qui ne retrouvera les joies de la lecture qu'après son opération oculaire (1351) n'y évoque pas une bibliothèque «orale ", ayant son seul siège dans la memoria, mais une collection de supports écrits. Il s'enthousiasme sur les poésies du moine Le Reclus de Molliens : «...Que moult volentiers, se pooie, / Les liroie trestous les jours $»^{6}$. À l'inverse, c'est dans un contexte fictionnel qu'est placée la bibliothèque, pourtant nullement imaginaire dans le détail, que l'on trouve au début du $\mathrm{XV}^{\mathrm{e}}$ siècle dans l'Inventaire des biens demourez du decés de l'amant trespassé de dueil de Pierre de Hauteville, le «Prince d'Amours» de la célèbre "Cour amoureuse» de Paris. Tout naturellement, les «classiques» de la poésie de l'amour courtois, comme le Lancelot $d u$ Lac et le Rommant de la Rose, figurent aux premières places de cette bibliothèque, conformément à l'un des objectifs essentiels de cette «Cour ", à savoir redonner vie aux idéaux chevaleresques et courtois. Cependant, on y trouve aussi des textes contemporains, tels que La Belle Dame sans Mercy d'Alain Chartier, Le Passe Temps de Michault Taillevent, L'Hôpital d'amour d'Achille Caulier et d'autres textes issus des 
cercles de la "Cour amoureuse» elle-même: la bibliothèque en tant qu'instance d'autolégitimation, en tant que memoria de ce qu'il convient de ne pas oublier mais que, au contraire, il ne faut cesser de rappeler, enfin en tant que lien entre le passé et un présent ouvert sur le futur.

5 Autre exception, la bibliothèque du pape Grégoire le Grand dans la Vie de saint Grégoire le Grand (1214) du moine Frère Angier, qui, en même temps, entame l'histoire des bibliothèques détruites dans la littérature française $e^{7}$. Ce n'est certes pas le tout premier exemple d'autodafé littéraire de livres que nous connaissions, lequel devrait être celui des livres de magie brûlés par des Ephésiens convertis par Saint Paul ${ }^{8}$, autodafé décrit dans les Actes des Apôtres 19,19 et invoqué jusqu'aux temps modernes en guise de justification de tous les bourreaux de livres. La bibliothèque de Grégoire le Grand, constituée par les écrits mêmes de son possesseur - et ce n'est pas par hasard si celui-ci apparaît fréquemment dans l'iconographie en tant qu'auteur tenant un livre ouvert, parfois en compagnie de son secrétaire ${ }^{9}$ - cette bibliothèque devient après sa mort la cible de ses adversaires romains, lesquels, ne pouvant plus s'attaquer à sa personne ${ }^{10}$, veulent anéantir tout ce qu'il a créé, pour le punir d'une famine dont ils le croient coupable: "Quant a son cors ne porent nuire / Ses oevres pristrent a destruire" (vv. 2793-2794 $)^{11}$. Le diacre Pierre, secrétaire et confident de Grégoire, réussit au moyen de son propre sacrifice assisté d'un miracle, à empêcher les pires conséquences de cette damnatio memoriae, et cela par la mise en scène d'une variante particulière de l'ordalie : au cas où, sous serment, il dirait la vérité concernant la sainteté de Grégoire ( deit estre de seinte memoire, / E entre les seinz anumbrez ", vv. 2848-2849) et la sagesse que renferment les écrits de Grégoire ( $Q$ Q'is deivent estre autorizez / Par tot lu mont de lonc en lez / Com cels qui sont seinz... » vv. 2857-2859), qu'il meure alors aussitôt après avoir prêté serment et qu'alors la destruction des livres soit interrompue ( des livres ardeir", v. 2869). Sinon, il coopérerait lui-même activement à l'autodafé ("Vos aidrai des livres ardeir», v. 2876). Il va sans dire que le secrétaire meurt. Par ce miracle patent («apert miracle», v. 2895), il prouve la véracité de ses paroles et sauve ce qui reste de la bibliothèque de Grégoire pour les générations futures qui, plus que jamais, en auront besoin.

6 Reste une certaine contradiction. D'une part, Pierre insiste sur le fait que la destruction de la «libraire», sacrilège, est en fait inutile puisque la gloire de Grégoire est, selon lui, définitivement acquise dans le monde entier. Les ouvrages du pape, dit-il, sont déjà largement répandus en plusieurs exemplaires, «objection déjà vérifiable chez des païens $»^{12}$. D'autre part et malgré cela, le secrétaire tient tout particulièrement à sauver la bibliothèque du pape. Cette contradiction peut être expliquée en premier lieu par l'hypothèse que Pierre le Diacre associe à la préservation de cette bibliothèque, en quelque sorte « auratique », la tentative de convaincre le «poeple» (v. 2828) de la sainte vérité déposée en ces écrits; seconde explication possible, le secrétaire essaie d'abord de détourner les adversaires du pape de leur projet à l'aide d'une ruse - puisqu'en définitive la reconnaissance de la sainteté de Grégoire perd son sens véritable si la vérité divine de ses écrits n'est pas conservée. Détruire la bibliothèque signifie en même temps taire et rendre inefficace la sagesse inspirée par Dieu. En brûlant le manuscrit, on ne brûle certes pas la vérité même dont il est le support, mais toutefois une copie précieuse de celle-ci. Il n'est pas étonnant que le caractère sacré du livre « unique » soit encore entier ici, à une époque des bibliothèques de manuscrits, dédiées au canon chrétien et dont chacune des composantes est, conformément à sa valeur 
spirituelle, réalisée materialiter avec un luxe littéralement unique, au titre d'objet sacré porteur de la parole divine (non seulement en tant qu' «écrit», mais aussi en tant que «livre»).

On en est encore au temps où l'exemplaire unique, dont la reproduction astreignante par des copistes est considérée comme un acte religieux, méditatif et donc à exécuter en silence, est la règle, et où la mise par écrit de la vérité est donc précaire. L'âge d'or du commerce du livre dans l'antiquité, lorsqu'un Tacite rapportait dans ses Annales (IV, 35) l'autodafé en public, décidé par le sénat romain et exécuté par les édiles, des livres de Cremutius Cordus et pouvait indiquer qu'ils survivraient dans nombre d'autres bibliothèques, cet âge d'or est depuis longtemps révolu. Et, d'autre part, la destruction par le feu d'une bibliothèque n'a pas encore acquis dans la conscience des gens de l'époque la signification d'un acte simplement symbolique, rituel qu'elle prendra après l'invention de l'imprimerie et l'essor massif de la production de livres. Pour le moment, la destruction de la bibliothèque (et non pas d'une bibliothèque parmi d'autres) demeure possible en principe - Victor Hugo en particulier rappelle toute l'importance de l'invention de Gutenberg inaugurant une ère nouvelle ${ }^{13}$. Il est encore possible, en détruisant une bibliothèque, de soustraire à l'humanité - en attente du Jugement dernier - la vérité divine incontestable dont elle a besoin. C'est là le motif du sacrifice de Pierre le Diacre: il est indispensable que le secrétaire de la vérité, intermédiaire subalterne qui a reçu de la bouche du saint le logos divin en tant qu'émanation de Dieu et du Saint-Esprit ("Por ço qe li seint Esperit / Lu espirot quanq'il [Grégoire] escrit », vv. 2913-2914) et l'a consigné par écrit («li ainz dit diacre Piere / Qi tant familier lu [Grégoire] iere, / Od qui lu Dialoge ainz dit [les quatre livres des Dialogi de Grégoire] / En desputant mist en escrit », vv. 2797-2800), ouvre les yeux pour cette vérité à ceux qui l'ignorent, en tant que confessor veritatis. De plus, «étant donné que Dieu accepte son [c.-à-d. celui du secrétaire] sacrifice en le laissant mourir, la relation tendue entre Dieu et les Romains est rétablie » ${ }^{14}$.

8 Mais une très grande partie de la bibliothèque de Grégoire, qui lui-même avait - d'après des assertions non confirmées de Jean de Salisbury - fait incinérer la Bibliotheca Palatina païenne, ou du moins les écrits astrologiques qu'elle renfermait ${ }^{15}$, a été réduite en cendres - en dépit de la croyance très commune au Moyen Âge que, par opposition aux écrits hérétiques, la Bible et les écrits théologiques orthodoxes résistaient au feu (ainsi qu'à l'eau) ${ }^{16}$. Rien d'étonnant donc à ce que les 34 derniers vers de ce texte hagiographique soient consacrés au constat du désastre :

Sachez por itant fut perie

Des livres la maire partie,

Car par l'arson furent perduz

Q'om ne seit q'is sont devenuz. (vv. 2915-2918)

De toute cette majeure partie de la bibliothèque ne subsistent que des traces indirectes - sous la forme d'une sorte de catalogue dressé par Grégoire lui-même, d'une «somme» (v. 2919), contenue dans un livre («escrit», «livre»), dans lequel figure la référence au compte-rendu écrit - de la main d'un élève - de commentaires oraux et de sermons du pape au sujet des Proverbes de Salomon, du Cantique des cantiques, des livres des prophètes, des livres des rois et de l'Heptateuque - un compte-rendu dont l'auteur précise cependant ne pas savoir ce qu'il en est advenu. S'ajoutent à cela d'autres écrits détruits avant d'avoir été lus («Ainceis qe ceus fussent seüs», v. 2948). Le 
regret de cette perte, qui, sans remettre en cause l'atteinte de l'entière vérité par l'exégèse des textes sacrés, risque tout de même de freiner la «vitesse » téléologique, est nettement exprimé.

Bien sûr, la bibliothèque d'auteur de Grégoire le Grand n'est pas, aux yeux du narrateur ou traducteur, une bibliothèque fictionnelle au sens propre - tout aussi peu que, de ce point de vue, le texte hagiographique et la vie de saint qu'il relate ne sont rangés dans la catégorie de l'imaginaire ${ }^{17}$. Elle souligne ainsi justement la signification fondamentalement religieuse que continuent d'avoir au haut Moyen Âge le «livre » et la «bibliothèque ». Le " centrage de la culture écrite sur le 'livre saint' $»^{18}$ entamé vers la fin de l'empire romain - en partant de l'élite intellectuelle chrétienne - n'est nullement révolu durant le haut Moyen Âge, même si la position «monopolistique» des livres sacrés, en tant qu'uniques points de référence d'une communication sociale globale orientée vers la vie éternelle, a subi des atteintes depuis le XII ${ }^{\mathrm{e}}$ siècle. La vérité attestée par le livre et la bibliothèque est d'essence divine, y compris dans le domaine du savoir séculaire, lequel demeure d'ailleurs, pendant le haut Moyen Âge, largement légitimé religieusement, même si c'est de manière de plus en plus indirecte. Et ceci ne vaut pas seulement pour la bibliothèque d'un prêtre que mentionne Philippe de Thaon dans la préface à son Comput (autour de 1120), en se référant à saint Augustin ${ }^{19}$. Ce n'est pas par hasard qu'au Moyen Âge le mot bibliotheca a non seulement le sens de «bibliothèque " mais peut également - jusqu'à son remplacement par biblia - avoir le sens, en tant que totum pro parte, de «Bible».

11 Le fait que la bibliothèque - avec son cœur, la Bible - se voit traditionnellement attribuer par les textes médiévaux le rôle de seul refuge de l'unique et absolue vérité, d'auctoritas, n'a donc rien d'étonnant. Et ceci justement, non pas dans des contextes fictionnels, mais essentiellement en relation avec l'assurance de vérité de la part de l'auteur dans le prologue ou l'épilogue. «E ço dit nostre armaries... » lit-on par exemple dans le Comput de Philippe de Thaon (v. 1727). Nombre de poètes mettent ainsi en rapport le "livre", source garante de vérité et modèle plein de science de leurs propres œuvres donc - livre généralement encore rédigé en latin pendant le haut Moyen Âge ${ }^{20}-$, avec la bibliothèque où il se trouve. Tandis que les vaniteux «fableor » (v. 1), les auteurs de fiction profane, ne présentent que mensonges à leur public- des informations de provenance orale, donc de transmission peu fiable, voire entièrement déformante -, Gervaise, auteur d'un bestiaire du début du XIII ${ }^{\mathrm{e}}$ siècle, fait référer son texte à un « livre» (en insistant sur l'authenticité de celui-ci : «ce n'est pas fable») dont il indique qu'il se trouve dans une bibliothèque, une armoire à livres («armaire», v. 34) ${ }^{21}$. La légitimation de l'affirmation de véracité est étayée par la localisation de cette bibliothèque à «Barbarie » (Barberie ou Barbery - abbaye cistercienne du diocèse de Bayeux) :

Gervases [...]

Vuet .j. livre en roman traitie[r].

Li livres a non Bestiaire.

Á Barbarie est [en] l'armaire

Li latins qui mult est plaisanz;

De illuec fu estraiz li romanz. (vv. 29-36)

Ce n'est que dans la mesure où l'auteur s'en tient à la vérité divine résidant dans la bibliothèque qu'il peut prétendre la transmettre à son public. 
13 Seul ce qui est issu de la bibliothèque des clercs est vrai. Même le perfectionnement moral de l'homme et l'entretien de sa crainte de Dieu ne sont possibles que grâce aux histoires de miracles puisées dans les «bons livraires» (v. 1) et les «bons livres» (v. 2) qu'ils renferment, nous dit Gautier de Coinci au début de ses Miracles de Notre Dame (après 1218) ${ }^{22}$. Il devient plus clair encore au début du prologue à la deuxième partie :

14 A Saint Maart ou biau livraire

Truis un biau livre dont biau traire

Vorrai encore bele matere

Et biaus mos de la bele mere

Le biau signeur de paradys. (vv. 1-5) 23

Ici aussi on remarque la localisation précise de la bibliothèque (le monastère SaintMédard à Soissons), ainsi que le rangement rhétorique de la «belle » bibliothèque dans une chaîne ascendante ininterrompue du beau - c'est-à-dire du bon - menant jusqu'à Dieu ou, plus exactement, prenant son départ en lui; enfin, il s'y ajoute une authentification plus que rhétorique, quasiment «autobiographique », car Gautier de Coinci était moine depuis 1193 et, depuis 1233, même prieur de Saint-Médard.

On trouve un passage similaire dans le prologue du recueil de miracles du moine et poète anglo-normand Adgar (ou Willame) ${ }^{24}$ datant de la seconde moitié du XII ${ }^{\mathrm{e}}$ siècle. Adgar lui aussi, qui considère la mention du nom de l'auteur comme devoir ( «Kar ne me uois mie tapir», v.19) et signal de véracité ("Par tant iert le liure sanz barate», v. 24), s'attribue la tâche de "translater " (v. 18) et indique - pour le répéter dans l'épilogue $e^{25}$ - le lieu de découverte précis de la vérité traduite par lui en langue du peuple :

16 Jo l'ai de saint Pol de l'almarie,

De saint Pol, de la noble iglise,

Ki en Lundres est bien asise. (vv. 40-42)

L'auteur anonyme d'une Vie de Sainte Euphrosine (XII ${ }^{\mathrm{e}}$ siècle), vraisemblablement basée sur un texte latin, ne fournit pas une indication aussi précise de l'endroit où se trouve son «livre»: «Eufrosine dame [...] En un livre d'armare vi escrite ta vie» (épilogue, vv. 1260-1262) ${ }^{26}$. Ici aussi cependant, c'est pour souligner la véracité de cette «Ystore bone et dulce, pleine de vérité» (v. 2) que le poète-adaptateur se sent appelé à faire connaitre à un plus large public, qu'il mentionne la provenance de la matière narrative emmagasinée, cachée et à (re)découvrir dans la bibliothèque.

Celui qui rapporte quelque chose en tant que témoin oculaire est digne de foi - déclare Jehan le Marchant dans le $18^{\mathrm{e}}$ "chapitre » de son Livre des miracles de Notre-Dame de Chartres $^{27}$. Il ne s'agit toutefois pas d'un véritable témoignage direct, c'est-à-dire d'une expérience de la réalité, mais de l'expérience textuelle de la lecture d'un texte latin, d'un « liures» en tant que source garantissant la véracité de ce qui y est rapporté («Que ce qu'il dit est chouse voire »). Le livre auquel se réfère Jehan provient de l'« aumoire » de la cathédrale de Chartres qui se trouve dans le «tresor $»^{28}$ de celle-ci,

Ou il a moult lectres et chartres

Preuileges de seignories

Et liures de toutes clergies

Qui melz vallent quargent ne quor.

Ce livre, dont l'extrême valeur matérielle (et donc spirituelle) est soulignée, a été trouvé dissimulé dans un bahut («Fou troue»- «Troue fu»), où il était enfermé 
inaccessible, oublié («Li liures qui nert pas seu / Ne il nestoit pas en memoire»). La (seule) vérité existe donc; il suffit de la trouver et - ainsi que l'explique l'auteur - de la rendre accessible par le biais de sa «traduction» dans la langue de la «laie gent», le «roumans", accessible à tous ceux qui ne connaissent pas le latin. Le contexte dans lequel le livre de vérité est situé souligne son caractère sacré, lequel le légitime également sur le plan du droit, mais montre en même temps la percée de "l'écrit pragmatique » (Hagen Keller) dans la bibliothèque, sans que pour l'heure soit remise en question la prédominance de la Bible et de ses dérivés, d'autant que les écrits mentionnés dans le texte de Jehan appartiennent visiblement à la «catégorie de luxe » administrative et du droit de propriété.

On ne s'étonnera pas, qu'outre la littérature édifiante, c'est-à-dire les textes se référant immédiatement aux livres sacrés, on trouve une attitude similaire dans la littérature profane. Par exemple, l'auteur de la chanson de geste tardive Baudouin de Sebourc

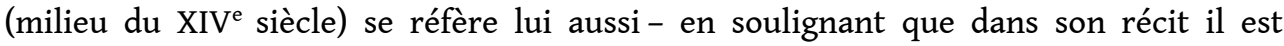
également question de «saintes » et de «saints» (v. 16) - à une bibliothèque en tant que source de sa «matère», pour authentifier une histoire de croisade plutôt profane, afin de prouver la véracité du récit en montrant son lien avec le lieu où sont conservés les saintes Écritures :

Ceste canchon, signour, doit bien estre prisie ;

Car translatez fu en divine clergie, Du Latin en Romans, n'el tenez à folie.

Á Saint-Amant, à Brugez, en la liberarie

En sont li fait escript. (vv. 1-5) ${ }^{29}$

La volonté d'inscrire cette chanson de geste dans la « divine clergie », en déclarant qu'il s'agit d'une traduction du latin, est censée être étayée par la localisation du prétendu modèle dans une bibliothèque de monastère. Le récit ( «li fait») n'est pas le fruit de l'imagination, n'est pas issu de la tradition orale du peuple mais de l'Écriture sainte gage d'authenticité. On retrouve le même phénomène - et là, le contexte sacré est bien plus justifié - à la fin de la Queste del Saint Graal: Gautier Map y indique que la transcription, qu'il aurait traduite en français, du «témoignage oculaire» latin de Bohort concernant les faits relatés serait conservée dans la bibliothèque du monastère de Salisbury:

21 Et quant Boorz ot contees les aventures del Seint Graal telles come il les avoit veues, si furent mises en escrit et gardees en l'almiere de Salebieres, dont MESTRE GAUTIER MAP les trest a fere son livre del Seint Graal por l'amor del roi Henri son seignor, qui fist l'estoire translater de latin en françois. ${ }^{30}$

22 La bibliothèque monacale en tant que sanctuaire de la vérité : le fameux prologue du Cligès de Chrétien de Troyes ne dit rien d'autre. Là, c'est la bibliothèque de la cathédrale Saint Pierre de Beauvais qui est la source et la garante de l'authenticitée ${ }^{31}$, mais plus remarquable que cette localisation ${ }^{32}$ est l'apologie générale du livre, au service de l'idée de la translatio studii, en tant que porteur et témoin de la vérité, laquelle vérité est présentée comme devant provenir de l'histoire, de la tradition (écrite), comme étant ainsi garantie par Dieu - vérité n'ayant jamais en fait à être ni «vérifiée » dans ses fondements, ni refaite et reformulée, mais tout au plus corrigée et complétée dans le détail : 
Qui tesmoingne l'estoire a voire;

Por ce fet de miauz a croire.

Par les livres que nous avons

Les fez des anciiens savons

Et del siecle qui fu jadis. (vv. 24-29)

Là aussi, la bibliothèque présente un caractère de Bible : elle fournit le témoignage écrit de la vérité inamovible en tant que fondement de la foi. Le fait qu'elle soit latine montre que la langue vulgaire - au contraire de la langue des écrits sacrés - doit encore lutter pour être reconnue comme garante de la vérité. L'inamovibilité de principe de la vérité est reflétée par la multiplication des livres et des bibliothèques dans les scriptoria. Le copiage permanent des textes faisant autorité - ainsi bien sûr que leur traduction dans la langue vulgaire - signifie la reproduction de la vérité dans le but de la propager, représente une muta praedicatio de la parole divine. Servir cette vérité équivaut à servir Dieu et à combattre l'Antéchrist. Le tot enim vulnera Satanas accipit, quot antiquarius Domini verba describit de Cassiodore est proverbial. La pratique de l'exégèse ne vise fondamentalement pas à élaborer une "contre-écriture » mais l'éclairage aussi exact que possible d'un texte sacré afin - compte tenu de la différence essentielle entre la langue humaine et celle de Dieu - d'en explorer jusqu'au dernier recoin ${ }^{33}$. C'est là également le but du mode de lecture de la ruminatio - en quelque sorte l'équivalent en matière de réception de la multiplication par les scriptores.

Mais la critique d'Homère dans le Roman de Troie (vers 1165) de Benoît de SainteMaure $^{34}$ montre que tout livre n'est pas porteur de vérité - du moins quand il ne fait pas partie de la bibliothèque chrétienne. Mais ici aussi, on trouve une histoire d'origine qui, cette fois-ci, débute dans une bibliothèque d'Athènes. C'est là en effet que Cornelius (Nepos), soi-disant neveu de Salluste, trouve par le plus grand des hasards alors qu'il cherche des livres savants en latin, le récit jusque-là perdu (vv. 116-120) de Darès :

A Athenes teneit escole.

Un jor esteit en un aumaire

Por traire livres de gramaire,

Tant $\mathrm{i}$ a quis e reversé

Qu'entre les autres a trové

L'estoire que Daire ot escrite... (vv. 86-91)

Il le traduit en latin (Historia de excidio Troiae), Benoît traduit la version latine conformément à l'idée de la translation - dans la langue romane. La vérité couchée dans le manuscrit, qui était cachée dans la cellule de base, la bibliothèque, et qui a dû de ce fait rester stérile ( $L$ Lonc tens fu sis livres perduz / Qu'il ne fu trovez ne veüz », vv. 117-118), tout en ayant par la même occasion été conservée, est à présent également à la disposition de ceux qui ignorent le latin, pour féconder le monde de l'esprit, en accord avec le devoir topique, mis en exergue dans le prologue, de transmettre le savoir et la vérité. en prose (XIII ${ }^{\mathrm{e}}$ siècle) :

$\mathrm{Si}$ vos ai ore menee a fin la veraie estoire de Troie selonc ce qu'elle fu trovee en l'almaire de saint Pol de Corrinte en grijois lenguaje, et dou grizois fu mise en latin et je la translatai en françois. ${ }^{35}$ 
Comme c'est le cas dans d'autres textes, ici aussi, la propagation et distribution du savoir par l'écriture présuppose la « découverte » de ce savoir dans une bibliothèque. Le fait que, dans le Roman de Troie, la bibliothèque chrétienne soit remplacée par une bibliothèque antique, est naturellement lié au sujet du roman, et se trouve plus ou moins déproblématisé par la légitimation chrétienne de la translatio. La récurrence de «trover» dans tous ces contextes saute aux yeux. À la différence des nombreuses réalisations $\mathrm{du}$ motif romanesque $\mathrm{du}$ manuscrit trouvé dans l'histoire littéraire postérieure, au Moyen Âge, le texte de base décisif est encore découvert, c'est-à-dire en fait remis en mémoire, dans le seul endroit où est emmagasinée la vérité - la bibliothèque. "Trover» au sens d'imaginer a pour préalable matériel et intellectuel «trover » au sens de trouver - puisqu'en définitive Dieu seul peut être un creator, peut créer du nouveau ${ }^{36}$.

On remarque par ailleurs que, dans la grande majorité de ces références à une trouvaille livresque et à l'endroit concerné, l'acte de trouver est corrélé à celui de la traduction. Comme le constate à juste titre Jacqueline Cerquiglini-Toulet ${ }^{37}$, les deux processus à l'origine de la production d'un texte en langue vulgaire sont des «ouvertures »: d'une part il y a l'ouverture spatiale de l'armoire à livres, de l'autre l'ouverture symbolique du livre - au moyen de sa transposition dans la langue vulgaire - à l'illettré, pour qui, autrement, la bibliothèque est doublement « fermée ». Le caractère élitaire de la bibliothèque en tant que bibliothèque cléricale est ainsi souligné une fois de plus. Encore au début de son Cymbalum Mundi (1537), l'humaniste Bonaventure Des Périers met en scène la même genèse textuelle :

Il y a huict ans ou environ, cher amy, que je te promis de te rendre en langaige françoys le petit traicté que je te monstray, intitulé Cymbalum Mundi, contenant quatre dialogues poetiques, lequel j'avoys trouvé en une vieille librairie d'ung monastere..$^{38}$

Et Guillaume Crétin souligne à la fois la grande importance de l'ouverture de la bibliothèque et celle du concept de la création poétique à partir de la bibliothèque quand, dans une complainte, s'adressant aux maîtres en la matière, il invite ceux-ci à se joindre à son deuil :

30 Abbé d'Auton, et maistre Jehan le Maire,

Qui est nostre sot estes des plus expers,

Ouvrez l'archet de vostre riche aumaire,

Et composez quelque plainte sommaire

En regrettant l'amy qu'ores je pers. ${ }^{39}$

Déjà une illustration datant du XIV siècle, dans un manuscrit enluminé du Roman de Troie de Benoît de Sainte-Maure ${ }^{40}$, représente le processus (d'ailleurs thématisé dans le prologue de ce roman antiquisant) de l'ouverture de la bibliothèque-armoire à livres : d'un geste expressif, deux lettrés offrent au lecteur du manuscrit l'accès à 14 codices de couleurs diverses, disposés sur quatre étagères. Le dos des livres est caché - suivant l'usage au Moyen Âge - ce que l'on pourrait comprendre comme invitation à ouvrir chaque livre à l'instar de l'armoire aux portes grandes ouvertes. Un jeune élève, sur la droite de l'image, a déjà répondu à cette invite : il lit dans un ouvrage ouvert. Au-dessus de la grande armoire, tout au milieu, se trouve encore une petite armoire murale à la porte également ouverte mais ne contenant qu'un seul livre, peut-être la Bible. Deux autres personnages - probablement un maitre et son disciple -, discutent apparemment de ce qu'ils ont lu. Au Moyen Âge, ouvrir l'armoire à livres était aussi essentiellement 
symbole d'accès à la mémoire, pour laquelle François Villon emploie encore la métaphore de l'armoire ${ }^{41}$.

31 En principe, la bibliothèque du Moyen Âge en tant que « collection de textes source » ne connaît pas de contradictions. Elle est d'un seul tenant. Chaque livre qui en fait partie la représente, et vice versa. Le centre de cette bibliothèque crée l'harmonie du vrai : c'est déjà, dans la description de la bibliothèque idéale par le bibliothécaire et «professeur de littérature » Cassiodore (Institutiones), la Bible, sans doute placée dans le premier armarium et englobant métaphoriquement la totalité du savoir humain ${ }^{42}$. Le livre des livres en tant que représentation (in persona) du Christ et la description du Christ avec des métaphores livresques ${ }^{43}$ soulignent ce fait. Hugues de Saint-Victor a comparé l'Écriture sainte à un "gigantesque corps de résonance, dans lequel chaque son tire sa signification de l'harmonie du tout $»^{44}$ : la Bible se situe dans la même relation d'harmonie vis-à-vis de la bibliothèque que chaque son biblique particulier par rapport à la Bible tout entière. Si dans l'imaginaire chrétien la construction de la tour de Babel a déjà montré le danger de la désharmonisation en matière de communication, la bibliothèque médiévale elle, en tant qu'institution collective, veut être en quelque sorte un instrument et un symbole de réharmonisation. Elle fait pratiquement figure d'« arsenal» contre les puissances du mensonge : claustrum sine armario, quasi castrum sine armamentario - c'est le jeu de mots attribué au chanoine Godefroy de Ste Barbe-enAuge (vers 1170) ${ }^{45}$. Si au bout du compte le monde est un livre que l'on peut déchiffrer, un texte cohérent, medium de la Révélation comme l'est l'Écriture sainte - et cela également parce qu'enfin toutes choses terrestres sont «en quelque sorte préconçues dans un livre transcendant $»^{46}-$, alors la bibliothèque elle-même, en tant que point de départ et point de fuite de la vie, ne peut, du fait de son centrage sur la Bible et ses dérivés, émettre un son discordant. C'est également dans ce sens qu'il faut entendre l'exigence faite aux scribentes dans un poème d'Alcuin, de mettre à la virgule près tous les signes de ponctuation au bon endroit et dans l'ordre (ordine suo) lorsqu'ils copient la Bible et les écrits des Pères de l'Église, afin de ne pas fausser le sens du texte, et surtout de ne pas «enrichir» l'original de leurs propres «frivolités »: hic interserere caveant sua frivola verbis ${ }^{47}$.

Ce ne sont donc pas seulement et pas primordialement le volume de l'explosion littéraire ni la démocratisation de la production de livres, qui, à la suite de l'invention de l'imprimerie - elle-même en grande partie conséquence d'un besoin de lecture progressivement accru - introduisent la pluralité dans la bibliothèque, et pas non plus uniquement la possibilité réduite de contrôle, essentiellement par l'Église, résultant de la naissance d'un marché du livre, lequel permet aussi la mise en relation de centres de savoir jusque-là isolés; en premier lieu, cette pluralité nouvelle est la conséquence de l'augmentation de la quantité du savoir et du bouleversement qualitatif par la sécularisation qui s'ensuit, de la décentralisation, de la libération de la bibliothèque de la prédominance des livres sacrés. Il est certes exact que le monde médiéval s'est scindé «en une multitude de petites, voire de minuscules, unités de communication qui n'étaient qu'insuffisamment reliées entre elles $»^{48}$. Toutefois, cette interconnection institutionnelle plutôt faible est largement déproblématisée par la bien plus efficace interconnection par le complexe des livres sacrés, voire par le mouvement d'extériorisation du centre de la mémoire et de la totalité du savoir en Dieu. Et au moment où, grâce à l'imprimerie, on faisait l'effort de restaurer l'unité du monde chrétien, qui, dans la réalité, devait plutôt être une non-prise de conscience de ses 
divergences potentielles préparées en particulier par la pratique de l'exégèse scolastique; à ce moment-là, c'était précisément l'écriture qui a en fait réellement permis la mise en évidence, la prise de conscience et le déploiement rapide de l'hétérogénéité, et surtout, ouvert la voie de la confessionnalisation. La mise en œuvre de l'imprimerie pour nombre de tentatives «réformatrices» de donner des normes au monde chrétien n'a pu avoir lieu qu'à partir du moment où la réalité de la pensée a déjà dépassé l'idée de l'unité. La globalisation des infrastructures communicationnelles par l'imprimerie n'a pu empêcher la « déglobalisation » croissante du savoir, mais l'a plutôt renforcée et accélérée.

33 La bibliothèque réellement plurielle et aux voix multiples, «la bibliothèque de Babel » et le processus de l'ouverture croissante et non plus seulement ponctuelle de la bibliothèque, ne commence de ce fait à exister - après quelques débuts hérétiques régionaux qui ne touchent cependant ni la théorie de la bibliothèque ni sa valeur symbolique - qu'à la Renaissance. À l'époque de Gutenberg, on reconnaît encore parfaitement la place centrale des livres sacrés au fait que la Bible et les textes parabibliques sont les premiers à bénéficier de la nouvelle technique de la production de livres. D'un autre côté cependant, un symptôme des plus nets de la perte de cette position-clé que vont désormais subir les textes bibliques est le fait que, il est vrai, la lecture et la possession de la Bible, essentiellement dans les milieux protestants, touchent des couches sociales de plus en plus basses, mais que justement, et pas seulement chez les illettrés, la Bible sort de plus en plus de la bibliothèque réservée désormais à des écrits profanes en premier lieu, pour trouver sa place à d'autres endroits de la vie quotidienne, tels que la cuisine. Par ailleurs, c'est à partir de la Renaissance que commence dans la fiction littéraire l'évolution progressive de la bibliothèque à la lecture univoque vers la bibliothèque contradictoire puis enfin illisible - une évolution qui reflète le processus analogue quant à la conception et la vision du monde et passe de surcroit par une séparation croissante de l'entité «livre des livres/Bible », facteur d'unité, et du « livre/bibliothèque de la nature » à l'acception de plus en plus contradictoire.

Certes, la mise en question de la bibliothèque harmonieuse, exempte de contradictions en tant que forme écrite d'une image du monde donnée par Dieu et reposant en lui, ne commence pas aux $\mathrm{XV}^{\mathrm{e}}$ et $\mathrm{XVI}^{\mathrm{e}}$ siècles. Cependant il reste relativement aisé pour «le sens de l'ordre » des «bibliothécaires » jusqu'à la fin du Moyen Âge, de neutraliser cette problématisation par une systématique apologétique. Un recours accru à la tradition normative du canon chrétien l'indique clairement. Ce recours, tout en étant incapable d'empêcher vraiment les déviations - peut-être même les favorise-t-il -, est cependant en mesure de les «récupérer » en quelque sorte, et de les «intégrer », de les conformer à l'ordre en les glosant. De cette manière, la bibliothèque de la réalité, jamais vraiment exempte de contradictions, peut être mise en conformité avec l'idée harmonieuse de la bibliothèque, tout comme la réalité de la Bible avec son idée : la Bible est identique à la vérité puisqu'elle contient la parole de Dieu, découle de Dieu; on ne peut cependant nier qu'elle ne contient pas que la vérité; cette contradiction peut être résolue par l'explication que Dieu est certes auctor de la Bible, mais que c'est l'homme qui est le compilator des vérités divines et responsable de ce qui, dans le texte biblique, ne correspond pas à la vérité et déforme la parole reçue ${ }^{49}$.

Les efforts pour conserver ce «bon» ordre chrétien apparaissent nettement aussi dans les « encyclopédies » latines puis en langue vulgaire du XIII ${ }^{e}$ siècle surtout ${ }^{50}$, copiées en 
de très nombreux manuscrits - substituts de bibliothèque généralement assez progressistes, dans la mesure où elles remplissent la fonction de remplacer pour un nombre croissant de lecteurs non-spécialistes le luxe rare d'une bibliothèque privée. En effet, ces Images $d u$ monde et Specula mundi, compilations du savoir contemporain consacrées aux res naturae et à la natura rerum, ne sont pas que des miroirs du monde, création de Dieu, "mis en ordre» et ainsi compréhensible d'une façon particulière, mais elles reflètent aussi le reflet de ce monde dans la bibliothèque, qui lui-même ne fait que refléter le logos divin. De ce fait, les tentatives de classification du savoir, l'« organisation» de toutes les connaissances, dans le cadre d'une vision systémique de la création essentiellement hiérarchique, sont tout à fait logiques - c'est ainsi seulement que les encyclopédies peuvent servir d' «aides à la lecture » à l'homme, appliqué à déchiffrer le livre de la nature dans lequel sont inscrites la bonté de Dieu, sa toute-puissance et la totalité de son savoir. Ceci explique par exemple l'ordre du haut (Dieu) vers le bas (la Terre) dans De proprietatibus rerum de Barthélemy l'Anglais. D'où il n'y a pas lieu de s'étonner que ces bibliothèques encyclopédiques insistent autant sur l'ordre en définitive cosmologique du savoir, compilé, extrait des "livres», que sur Dieu, l'origine de cet ordre ${ }^{51}$ qui en principe réclame aussi une lecture "ordonnée ", bien que dans une certaine mesure une lecture sélective, fragmentaire, pragmatique soit déjà possible ${ }^{52}$. Des signaux de structuration dans les manuscrits (sommaires, rubriques, initiales et miniatures) soulignent l'agencement du livre encyclopédique, qui reflète l'ordre du monde, et servent à l'orientation du lecteur. Sans mettre en cause l'ordre harmonieux de l'ensemble, ces signaux qui mettent en évidence un tel ordre permettent en même temps de se concentrer sur des éléments isolés, de s'approprier leur vérité partielle, mais dans l'assurance que celle-ci ne possède pas d'autonomie et reste en permanence reliée à l'intégralité de la vérité de la révélation chrétienne. Cependant, la vérité de ces bibliothèques encyclopédiques, de ces summae, demeure vérité de bibliothèque, dans la mesure où elle ne se présente que comme issue de la compilation de la bibliothèque même.

Mais sans aucun doute, cette conception particulière de la vérité - la vérité en tant que vérité reproduite - est liée au degré élevé d'intertextualité, au caractère autoréférentiel de la littérature médiévale, au-delà de la seule littérature courtoise. Aussi rares que soient les bibliothèques fictionnelles dans la littérature du Moyen Âge, aussi souvent celles-ci se présentent comme bibliothèques d'intertextes. Nous savons d'ailleurs que cette tendance à l'intertextualité s'accroît considérablement au XIV ${ }^{e}$ siècle. La pratique du jeu avec le topos se répand. Le degré accru d'autoréflexivité et de référence à des modèles littéraires des XII ${ }^{e}$ et XIII ${ }^{e}$ siècles fait que la littérature de la fin du Moyen Âge devient quasiment une « littérature au second degré $»^{53}$, dans laquelle les réminiscences littéraires passent au premier plan. C'est dans ce contexte qu'il faut voir une métaphore inhabituelle de la bibliothèque : la bibliothèque-cimetière. En 1457, dans son Livre du Cuer d'Amours espris, René d'Anjou a imaginé un tel «cimetière» des classiques de la littérature amoureuse (Ovide, Jean de Meun, Boccace, Guillaume de Machaut, Pétrarque et Alain Chartier) ${ }^{54}$.

On voit d'ailleurs toute l'importance qu'avait pour le Moyen Âge chrétien la bibliothèque prépluraliste, harmonieuse et exempte de contradictions en tant qu'idée au milieu de la multitude - croissante depuis la fin de l'antiquité, attestée et illustrée par nombre de textes historiographiques et légendaires-, de destructions de bibliothèques, en particulier par le feu ${ }^{55}$. Celles-ci sont particulièrement 
symptomatiques quand, dans certains cas, les récits qui les transmettent ne reposent pas sur des faits historiques. C'est d'elles justement qu'on avait besoin pour maintenir pure la bibliothèque « imaginaire » après le «tournant constantinien ». Dans un certain sens, les autodafés de livres et de bibliothèques avant l'Inquisition, et sous celle-ci, constituaient également une réponse à la persécution de la religion chrétienne, basée sur le livre, les saintes Écritures, par l'Empire romain, surtout suite à l'édit promulgué par l'empereur Dioclétien (303). Le caractère rituel de ces autodafés de livres est conforme à leur fonction purificatrice. Les décrets en la matière, épiscopaux, synodaux voire conciliaires, étaient légion. Le nombre relativement restreint d'écrits hérétiques et schismatiques qui nous sont parvenus s'explique par là. Un reflet artistique de cette forme de protection de la bibliothèque chrétienne orthodoxe est par exemple encore discernable dans la fresque plafonnière de Matthäus Günther à Inckersdorf, La gloire de saint Augustin, dont les livres émettent des éclairs qui vont frapper le groupe sombre des hérétiques et mettent le feu à leurs écrits, ou encore sur un poêle rococo exposé au musée Carolino-Augusteum de Salzbourg qui présente en son milieu une banderole portant les mots Bibliotheca Vulcano consecrata, et sous laquelle les carreaux de faïence forment les dos d'un grand nombre de livres, écrits hérétiques de l'antiquité, du Moyen Âge et de l'époque moderne - ainsi livrés au feu purificateur du poêle ${ }^{56}$.

L'iconographie médiévale est moins "criarde» mais tout aussi éloquente. Ne mentionnons qu'un seul exemple: le manuscrit 59 de la bibliothèque de la cathédrale de Cologne, qui recèle surtout un certain nombre de lettres de saint Jérôme, présente une miniature couvrant toute la première page $\left(1^{r}\right)^{57}$. Sur la moitié inférieure est représenté le commanditaire du manuscrit, l'archevêque de Cologne Frédéric $\mathrm{I}^{\mathrm{er}} \mathrm{de}$ Schwarzenburg (1100-1131), dans une pose qui est censée le caractériser comme se situant dans la succession de saint Jérôme et veillant à la conservation de ses écrits. Inspiré par l'esprit de Jésus trônant au-dessus de lui, il médite sur la loi divine - la banderole qu'il porte cite le psaume 118, 97 (Quomodo dilexi legem tuam Deus tota die meditatio est). L'agencement est éloquent: la vérité vient de Dieu en passant par le Christ et arrive jusqu'à l'archevêque trônant sur le faldistoire et responsable de la conservation et de la propagation de cette vérité - la banderole du Christ déclare si quis diligit me, sermonem meum servabit (Jean 14,23). La loi divine, la vérité, apparaît, pour que le message soit bien clair et pour souligner la typologie de saint Jérôme, matérialisée dans la bibliothèque de Frédéric, sous la forme de deux caisses de livres placées à droite et à gauche de lui et vers chacune desquelles, détail significatif, est tournée une des têtes de lion du faldistoire. Le nombre des livres et leur répartition harmonieuse n'ont rien de surprenant: douze livres, six par caisse, chaque volume à l'intérieur d'une caisse étant de couleur différente mais ces couleurs étant les mêmes dans les deux caisses. Les hexamètres entourant l'ensemble de la miniature soulignent de surcroît la fonction de lieu de stockage et de mémoire de cette bibliothèque, en évoquant surtout la «ruminatio»:

...quo nova lex promit, libris et corde recondit,

hinc dulci gustu, quod ruminat, exhibet actu.

On trouve une espèce de résumé (très bibliophile) de l'idée de la bibliothèque dans le Moyen Âge chrétien, dans le Philobiblon de Richard de Bury, que cet homme d'État, savant et littérateur anglais, évêque de Durham depuis fin 1333 et mort en 1345, a écrit au cours des deux dernières années de sa vie ${ }^{58}$. Aucun doute : tout savoir et toute vérité sont fondés en Dieu, le liber vite (77). Elle, cadeau de la générosité divine envers les 
hommes, est de ce fait une sagesse éternelle, immuable, que le temps historique ne peut entamer. Étant donné l'imperfection de la transmission purement orale de la vérité, et surtout le caractère éphémère de la parole, Dieu - en totale méconnaissance du célèbre jugement platonicien dans le Phaidros, selon lequel l'écrit est nuisible à la mémoire - a fait fixer le tabernacle de sa vérité dans les livres, considérés quant à eux comme gardiens fiables et pérennes de la mémoire. Donc, le savoir repose dans les livres, mines profondes de la vérité (profundissime sophie fodine, 79). L'harmonie de la bibliothèque telle que Richard de Bury la conçoit, est absolue: Per libros preteritorum reminiscimur, de futuris quodam modo prophetamus, presentia que labuntur et fluunt scripture memoria stabilimus (121). Le passé (souvenu), le présent (identifié) et le futur (eschatologiquement assuré) peuvent être intégrés en son sein en un agencement cohérent du savoir, en un ordre qui ne peut être mis en question du fait que tout est orienté vers un centre unique et incontesté - les "livres sacrés»: veritatem vero potissime et tuentur et continent sacri libri, immo sunt veritas ipsa scripta (80). L'acte de commenter les écrits sacrés n'ajoute au fond aucune vérité nouvelle, mais se contente de mettre celle-ci au jour. Les seules carences inhérentes à la bibliothèque sont dues à l'homme et à son imperfection: il ne s'agit pas là en premier lieu des dangers, abondamment décrits par Richard de Bury, qui menacent la bibliothèque tels que les guerres ${ }^{59}$ ou le manque de bibliophilie, surtout de la part des Ordres Mendiants, mais de la conséquence immédiate de la construction de la tour de Babel : bene graciosa fuisset librorum condicio, si turris Babel nullatenus obfuisset presumptio, si totius humanis generis unica descendisset sermonis species propagata (88-89). Car la propagation, devenue nécessaire après Babel, des livres sacrés au moyen de leur traduction dans une autre langue ( $\mathrm{du}$ grec en latin, du latin dans la langue vulgaire) ${ }^{60}$ - conformément à la translatio studii -, ou de leur commentaire (en langue étrangère également), recèle le grand risque que leur vérité soit déformée. Mais pour autant, tout ce que comprend la bibliothèque, que par ordre d'importance Richard de Bury range immédiatement après les reliques du Christ, n'en reste pas moins irremplaçable vérité du salut :

Patres igitur reverendi, patrum vestrorum dignemini reminisci et librorum propensius indulgete studio, sine quibus quelibet vacillabit religio, sine quibus ut testa virtus devocionis arescet, sine quibus nullum lumen poteritis mundo prebere (91)

Bien sûr, à l'exhortation à prendre soin de la bibliothèque déjà existante en tant que siège de la sagesse, à la commenter et à la copier sans arrêt - selon lui copier des livres sacrés est la plus noble tâche dont l'homme puisse s'acquitter -, Richard de Bury ajoute le devoir envers Dieu de créer de nouveaux livres. On ne s'étonne cependant pas que priorité soit donnée aux « anciens » : pour l'auteur du Philobiblon, une véritable Querelle des anciens et des modernes est hors de question. Certes, les «modernes» sont tout à fait en mesure d'ajouter aux phrases des Pères quelque chose d'utile et de bon, mais aux yeux de Richard de Bury, qui cite à cet effet le grammairien Phocas, objet de nombreux commentaires au Moyen Âge, dans son Ars de nomine et verbo (autour de 400) :

41 Omnia cum veterum sint explorata libellis, multa loqui breviter sit novitatis opus (105) ${ }^{61}$

Par ailleurs, ce bibliophile du Moyen Âge souligne que la qualité de la bibliothèque de son époque est le résultat d'un travail collectif depuis le début de l'humanité pensante, travail progressif auquel chacun n'a apporté qu'une infime partie, et qui ne réside pas uniquement dans l'ajout de connaissances nouvelles-plus exactement de connaissances nouvellement découvertes -, mais aussi dans l'élimination d'erreurs. Et à 
ce sujet l'auteur cherche moins à insister sur la valeur de l'erreur, dans la perspective de la métaphysique aristotélicienne, qu'à caractériser l'histoire du savoir comme processus continu de perfectionnement mu par l'imitatio et l'aemulatio - processus dont les analogies structurelles avec la formulation de l'idée de la bibliothèque de Victor Hugo dans Notre-Dame de Paris ne sont limitées que par le fait que pour le «romantique classique ", le concept d'un progrès qui ne s'arrête pas au présent est primordial, alors que pour l'auteur du Philobiblon, la progression même dans la perception de la vérité donnée est déjà, pour l'essentiel, histoire - dans la transition du paganisme au christianisme. La bibliothèque apparaît comme un point final dont la propre genèse lui est en même temps inhérente sous la forme des innombrables inter vetustissimos et novellos intermedii :

Quid fecisset Vergilius, Latinorum poëta precipuus, si Theocritum, Lucretium et Homerum minime spoliasset et in eorum vitula non arasset? quid nisi Parthenium Pindarumque, cuius eloquentiam nullo modo potuit imitari, aliquatenus lectitasset? Quid Sallustius, Tullius, Boëtius, Macrobius, Lactantius, Martianus, immo tota cohors generaliter Latinorum, si Athenarum studia vel Grecorum volumina non vidissent ?... (110)

Revenons à la question implicite du début: pourquoi la littérature de fiction médiévale connait-elle si peu le motif de la bibliothèque ? Naturellement, dans une culture en voie de transition - sur le plan culturel global et non pas uniquement du point de vue clérical - de l'oralité dominante vers la domination de l'écrit, le lieu de stockage de la mémoire collective qu'est la bibliothèque ne joue encore qu'un rôle plutôt modeste surtout dans le domaine de la langue vulgaire - même si après 1300 , ce processus est déjà bien avancé en raison entre autres du passage de la poésie chantée à la poésie lue, et de la présentation orale de livres à leur lecture. Certes, c'est au XIV e siècle que débute l'histoire de la bibliophilie esthétisante, mais elle demeure d'abord limitée à une élite de hauts dignitaires. Certes aussi, aux $\mathrm{XIV}^{\mathrm{e}}$ et $\mathrm{XV}^{\mathrm{e}}$ siècles l'écrit et le livre connaissent un gain de considération sensible par rapport à la memoria - ils ne sont plus dès lors considérés comme nuisibles à celle-ci (comme c'est encore le cas chez Pétrarque), mais comme ses serviteurs, par le fait qu'ils permettent l'expression de ce qui est stocké dans la mémoire; cependant, ce n'est qu'à la Renaissance que cette évolution s'affirmera pleinement.

De manière générale, on peut donc dire que la rencontre avec le livre conservera longtemps encore un caractère d'exception. Sur le plan oral de la littérature en langue vulgaire, c'est encore essentiellement la mémoire de ceux qui récitent la poésie qui tient lieu de bibliothèque, même si cette production littéraire tire ou bien prétend tirer ses origines de la bibliothèque en tant qu'entité supérieure, du «livre». Les bibliothèques individuelles, lesquelles présupposeraient un stade de pratique de la lecture beaucoup plus avancé que ce qu'il apparaît être même dans l'alphabétisation progressive et l'importance croissante de l'écrit pragmatique depuis le XII ${ }^{e}$ siècle, sont rares: ce n'est que depuis la seconde moitié du XIII ${ }^{e}$ siècle et dans le milieu ecclésiastique que leur nombre et leur taille augmentent, et parmi elles, celle de Richard de Fournival devrait avoir été l'une des toutes premières grandes bibliothèques privées. Le répertoire des plus grandes bibliothèques religieuses du Moyen Âge dépasse rarement le nombre de 300 volumes. Les bibliothèques collectives ne sont pas accessibles au public, l'extériorisation de la mémoire emmagasinée dans les bibliothèques est très loin d'avoir atteint son point culminant. L'expérience du livre et de la bibliothèque demeure donc restreinte. La bibliothèque est présente bien 
davantage en tant qu'idée qu'elle ne l'est en tant que réalité. Et en tant qu'idée, elle est homogène, centrée sur la Bible, pratiquement synonyme des saintes Écritures et à ce titre autorité indiscutée, source et destination de chaque livre, gardienne de la vérité pour laquelle il n'existe pas d'affirmation d'originalité individuelle mais à laquelle revient une authentification essentiellement intertextuelle. Dans cette mesure, le caractère autoréférentiel de la littérature médiévale - qui tout en étant indéniable n'est quand même pas absolu et doit être complété par la référence à la bibliothèque de la nature - implique finalement aussi une référence à l'idée de la bibliothèque chrétienne dont elle découle et dans la tradition de laquelle elle s'inscrit. Peut-être même pourraiton voir - toutes proportions gardées - dans le concept de la "poésie formelle» de Robert Guiette ${ }^{62}$ une analogie esthétique à cette orientation marquée de la bibliothèque sur un centre dont en principe le rayonnement n'autorise guère que des reformulations et des variantes au niveau de l'expression, mais aucun véritable franchissement ou affranchissement. Bien entendu, la subversivité existe dans le cadre global de cette littérature. Mais vu qu'elle ne remplit pour l'essentiel qu'une fonction carnavalesque, compensatoire au sens large, elle n'est pas réellement en mesure de remettre en question l'idée de la bibliothèque - une remise en question qui représente un préalable important pour sa fictionnalisation. Mais ce changement-là n'interviendra qu'à partir du XVI siècle.

Il va de soi que les textes de nature séculaire ne sont pas à priori qualifiés de subversifs. On sait que la compatibilité des artes liberales issues du système scolaire de la fin de l'antiquité avec la doctrine chrétienne a été pour l'essentiel atteinte dès la fin de l'antiquité et n'est plus mise en doute que sporadiquement au Moyen Âge. La nécessité de connaître ces artes pour pouvoir étudier la théologie ou la philosophie n'a jamais vraiment été contestée. La bibliothèque que, dans la première moitié du $\mathrm{V}^{\mathrm{e}}$ siècle, le païen Martianus Capella fait vomir à la très savante vierge laïque Philologia avant son apothéose et en tant que préalable purificateur à son immortalité (De nuptiis Philologiae et Mercurii, livre II), s'est imposée partout au Moyen Âge, sous une forme modifiée. La bibliothèque riche de quelque 300 codices de Richard de Fournival - de l'avis général sans doute plutôt une bibliothèque idéale imaginée qu'une bibliothèque réelle -, dont le catalogue a été dressé par l'auteur du Bestiaire d'Amours au milieu du XIII ${ }^{\mathrm{e}}$ siècle dans sa Biblionomia ${ }^{63}$, se caractérise par exemple en particulier par le fait que des trois areolae qu'elle contient, la médiane - située entre philosophia (y compris les artes liberales) et la theologia - est dédiée aux lucrativae scientiae. Les deux groupes principaux de cette areola représentent d'une part le droit canon et le droit séculaire - pour Richard de Bury encore, les ouvrages de droit, en tant qu'écrits non scientifiques, sont en fait indignes de figurer dans une bibliothèque -, d'autre part la médecine, dont la position prééminente est certainement liée à la profession de médecin du père de Richard de Fournival, profession que lui-même aussi exerça un temps. Mais même dans la bibliothèque de Richard de Fournival, introduite par une peu originale métaphore de jardin, l'ordre hiérarchique des areolae est nettement marqué : la cote des livres consiste en lettres de caractères et de couleurs différents. Les lettres dorées sont réservées à la theologia (plus précisément à la Bible et ses commentaires) : areole tamen sacram continenti scripturam color litterarum aureus specialiter reservatur (521).

Une position analogue ressort déjà des Versus in bibliotheca d'Isidore de Séville ${ }^{64}$ (début du $\mathrm{VI}^{\mathrm{e}}$ siècle). Même dans la bibliothèque de cet "encyclopédiste", dont on sait la sévérité envers la lecture d'ouvrages profanes, les textes laïques ont droit de cité, tels 
les ouvrages de poètes chrétiens, d'historiens ecclésiastiques, et enfin ceux de juristes. En outre, dans le titulus X consacré aux auteurs chrétiens, les auteurs païens Virgile, Horace, Ovide, Perse, Lucain et Stace sont certes mis en contraste avec Prudence et ses «collègues », mais dans l'ensemble mentionnés de manière si neutre qu'on ne saurait en déduire une condamnation globale des auteurs de l'antiquité romaine. Cela correspond parfaitement au titulus introducteur des Versus in bibliotheca :

Sunt hic plura sacra, sunt mundialia plura;

Ex his si qua placent carmina, tolle, lege.

Prata vides plena spinis et copia floris;

Si non vis spinas sumere, sume rosas.

Il est permis de supposer que les combinaisons mundialia-spinae et sacra-rosae impliquent des jugements de valeur.

Même si l'idée de la bibliothèque au Moyen Âge présente un caractère relativement unitaire, à la fin du Moyen Âge on discerne cependant déjà les premiers signes de désagrégation et de changements de statut. Sur ce point apparemment, le langage des signes de l'iconographie est plus éloquent - parce que moins explicite et ainsi plus libre - que la langue écrite. Pour cette raison, nous nous proposons pour finir de mentionner au moins deux cas tirés de l'art figuratif autour du $\mathrm{XV}^{\mathrm{e}}$ siècle.

Le premier, aux occurrences multiples, concerne l'art funéraire ${ }^{65}$. Quand les personnages représentés sur les monuments funéraires du haut Moyen Âge tiennent en mains un livre (souvent ouvert), c'est pour les pourvoir d'un signe de piété - tout comme c'est le cas en particulier pour la représentation de saints et d'ecclésiastiques dans les enluminures. C'est particulièrement quand les personnages ne lisent pas ces livres mais ont les yeux clos (Éléonore d'Aquitaine dans l'abbaye de Fontevrault), ou quand il pressent le livre (généralement) fermé contre leur poitrine, que la symbolique religieuse devient évidente. Dans tous ces cas il s'agit d'un «livre sacré» représentant la bibliothèque dans son ensemble. Au XVe siècle cependant, on note un changement. $\mathrm{Au}$ livre en tant que symbole de la sagesse divine et en tant que réconfort sur le chemin de l'au-delà, s'ajoute de plus en plus le livre en tant que symbole du savoir séculaire, de la vie intellectuelle en général. Ceci a deux conséquences. D'une part, le nombre des livres s'accroît, parfois de façon considérable. Du livre (unique), pars pro toto d'une bibliothèque unitaire, on passe petit à petit à la bibliothèque pluraliste, polyphonique. La pierre tombale du juriste et pasteur Magnus Schniechen (église Saint-Jacques à Straubing), mort en 1418, est encadrée de livres en haut, à droite et à gauche de l'oreiller, les pieds reposent sur trois in-folio et un autre livre (ouvert) est placé sur la poitrine. On retrouve cet agencement sur nombre de pierres tombales et de tombeaux de l'époque. D'autre part, il apparaît clairement que dans tous ces exemples, les livres sont mis en relief en tant que symboles de l'érudition et de la sagesse essentiellement humaine. Autrement dit: ces livres sont des insignes de l'activité professionnelle des défunts, donc de leur érudition spécifique, acquise de leur propre effort. Sous la bière du gisant de l'évêque Orso (mort en 1511) à San Marcello al Corso (Rome) par exemple, on voit un impressionnant amas de livres - Orso était un amateur de livres qui en aurait rassemblé plus de 700 .

Le second cas concerne surtout la peinture à fresque de la fin du Moyen Âge. Celle où le peintre véronais Altichiero da Zevo (seconde moitié du XIVe siècle) a représenté Pétrarque dans la Sala dei Giganti de Padoue ${ }^{66}$, montre, dans sa version originale dont on peut voir la reproduction dans une miniature illustrant De viris illustribus dans un 
manuscrit (ms. 101) de la Hessische Landesbibliothek à Darmstadt, le poète seul dans son "studiolo", coupé du monde, entièrement consacré à l'étude. Il est assis le buste droit et dans une position surélevée sur une chaise à dossier, sorte de trône du poète couronné ; sur sa table de travail se trouve un petit pupitre supportant un livre ouvert qu'il lit méditativement - à côté, sont disposés du papier, de quoi écrire et un encrier. Le poète est entouré de ses livres - environ trente au total, c'est-à-dire un nombre plutôt considérable. Ces codices se trouvent pour la plupart à l'arrière-plan où ils reposent sur deux étagères dans une armoire ouverte. Fritz Nies interprète à juste titre l'agencement de cette image en tant que symbole de la création poétique: de la bibliothèque inspiratrice au nouveau livre. Rien n'évoque le monde extérieur. Il y a certes à l'arrière, à gauche de la bibliothèque, une petite fenêtre - mais juste devant elle se trouve une "roe d'estude $~^{67}$ qui la cache à moitié - et de plus on ne discerne rien à travers. Nous voyons une pièce entièrement fermée dans laquelle ne circule que l'esprit de la bibliothèque médiévale - personnifié par Pétrarque en tant que saint Jérôme sécularisé.

50 La modification au $\mathrm{XVI}^{\mathrm{e}}$ siècle de cette fresque diffère considérablement de la version primitive : la bibliothèque à l'arrière-plan a disparu, remplacée par une vaste ouverture sur un impressionnant paysage de montagne avec au premier plan un moulin à eau. Qu'il faille ou non voir ici une allusion de l'artiste à l'importance des paysages dans la poésie de Pétrarque voire même à son ascension du mont Ventoux : «le Pétrarque revu et corrigé par le XVI ${ }^{e}$ siècle ne sera toujours pas dépourvu de livres, mais visiblement ce n'est plus des lectures seulement qu'émanera le souffle créateur qui l'anime $»^{68}$. La bibliothèque réduit sa référence à elle-même. L'unique vérité, divine, fait place à une nouvelle vérité venue de l'extérieur, sans devoir pour autant être tout à fait remplacée par cette dernière.

51 L'examen d'une étape intermédiaire, une miniature représentant Pétrarque (dans un manuscrit des Rime e Trionfi) ${ }^{69}$ et datant du $\mathrm{XV}^{\mathrm{e}}$ siècle, montre qu'il s'agit là du résultat d'un processus progressif: ici aussi, Pétrarque est assis dans son «studiolo" sur un banc devant son pupitre; devant lui sont disposés environ vingt livres sur cinq étagères, l'un d'entre eux ouvert face au poète représenté dans la position du penseur (joue gauche appuyée sur la main, les yeux ouverts) et en train de coucher des vers sur une feuille de papier. À l'arrière-plan on voit une petite fenêtre, une espèce de meurtrière percée dans la muraille et qui en dépit de ses dimensions modestes sert de point de fuite à toute la scène construite d'après les loix de la perspective, fonction que soulignent les couleurs vives (vert, jaune, bleu) de la nature que l'on aperçoit à travers cette ouverture. Mais dans cette miniature également, Pétrarque se détourne encore du monde extérieur en lui tournant le dos. En revanche, sur la fresque (remaniée) du XVI ${ }^{\mathrm{e}}$ siècle, sans fenêtre, le regard est totalement libre, et Pétrarque n'a plus qu'à tourner la tête voire même seulement les yeux légèrement vers la gauche pour pouvoir contempler sans limites le monde extérieur. Le monde, le livre du monde est ouvert. Sa relecture par l'homme, qui ne veut plus se satisfaire de la lecture divine du monde, peut commencer. Ainsi, la bibliothèque cesse d'être avant tout un lieu de stockage rétrospectif. Elle va maintenant de plus en plus prendre un caractère prospectif.

On peut considérer comme symptôme du point culminant de cette évolution au XVI siècle une célèbre description littéraire d'un "studiolo»: il s'agit de celle que Montaigne fait de sa propre «librairie» dans ses Essais (III, 3) : dès le début de la présentation, il signale la solitude et la conscience élitaire - l'individu se trouve seul, au 
troisième et dernier étage d'une tour ${ }^{70}-$, mais aussi la configuration ouverte du lieu : «ma librairie, d'où tout d'une main je commande à mon ménage. Je suis sur l'entrée et vois sous moi mon jardin, ma basse-cour, ma cour et dans la plupart des membres de ma maison». Ici s'imbriquent clairement la réception (non systématique) de la bibliothèque par la lecture ( «Là, je feuillette à cette heure un livre, à cette heure un autre, sans ordre et sans dessein, à pièces décousues »), la méditation (" tantôt je rêve ») et la production (tout aussi peu systématique) des Essais («tantôt j'enregistre et dicte, en me promenant, mes songes que voici»). La flânerie intellectuelle, qui pourrait être favorisée encore davantage par des modifications architecturales, n'est pas seulement nourrie par les livres disposés sur cinq étagères qui font le tour de la pièce et que le regard peut embrasser « d'une vue» de la table de travail, mais aussi par la promenade des yeux. Car cette pièce en haut de la tour « a trois vues de riche et libre prospect».

À l'ouverture de l'armoire à livres au-delà de la mince couche des élites cléricales, étape qui n'est nullement achevée au temps de Montaigne et qui même au $\mathrm{XVI}^{\mathrm{e}}$ siècle est encore loin de se faire sans résistances ${ }^{71}$, s'ajoute l'ouverture au monde des couches cultivées. L'érudition livresque ne se nourrit plus seulement d'elle-même mais de plus en plus de l'expérience du monde, de la perception sensorielle de la nature, de la lecture directe dans cette dernière. Ce qui au XVI ${ }^{e}$ siècle peut parfois apparaître comme «mentalité anti-littéraire » n'est en fait que la correction qui commence à se faire sentir, de la domination scolastique du canon du savoir emmagasiné dans la bibliothèque. Selon le médecin Paracelse, partisan de l'examen naturae, l'expérience directe de la réalité est à préférer à tout ce qui est lu et écrit. Mais la valeur de la bibliothèque de livres n'en est en rien diminuée, puisque la lecture du monde lui est ensuite incorporée pour l'enrichir et pour lui conférer ce dynamisme qui fait défaut à la bibliothèque scolastique.

\section{NOTES}

1.On trouve cette double définition déjà dans De significatione verborum de Sextus Pompeius Festus (seconde moitié du II siècle).

2.Pour ce qui est du Moyen Âge, voir surtout André Vernet (éd.), Histoire des bibliothèques françaises. Les bibliothèques médiévales, Paris, 1989.

3.Voir par exemple les célèbres galeries satiriques de poètes chez les troubadours Peire d'Alvernha (P.-C. 323, 11) et Moine de Montaudon (P.-C. 305, 16), ou encore les «sirventes-ensenhamens » des troubadours Guiraut de Cabreira, Guiraut de Calanson et Bertran de Paris. Dans sa chanson P.-C. 242a, 1, Guiraut de Cabreira par exemple présente à son jongleur Cabra presque l'ensemble du répertoire du parfait jongleur quasiment sa bibliothèque - pour le domaine linguistique occitan-français vers le milieu du XII ${ }^{e}$ siècle.

4.Une liste assez longue d'œuvres présentées par des jongleurs se trouve dans Flamenca. Concernant cette sorte de «bibliothèque orale ", voir notre article « Par devant lui chantent li jugleor'. La poésie médiévale dans le contexte du 'Gesamtkunstwerk' » du 
repas courtois, dans : Dietmar Rieger, Chanter et Dire. Études sur la littérature du Moyen Âge, Paris, 1997, pp. 89-110.

5.Éd. Wendelin Foerster, Wien 1874.

6.Gilles li Muisis, Poésies, éd. du baron Kervyn de Lettenhove, Louvain, 1882, tome I, pp. 86-87.

7.On trouve la représentation picturale d'un autodafé de livre dans le lai Guigemar de Marie de France : la décoration murale de la pièce dans laquelle le châtelain jaloux a enfermé son épouse montre l'incinération par Vénus des Remedia Amoris d'Ovide (vv. 239-241).

8.Voir à ce sujet Hermann Rafetseder, Bücherverbrennungen. Die öffentliche Hinrichtung von Schriften im historischen Wandel, Wien-Köln-Graz 1988 (Kulturstudien. Bibliothek der Kulturgeschichte 12), pp. 39-40 ; Wolfgang Speyer, «Büchervernichtung », Jahrbuch für Antike und Christentum, 13, 1970, pp. 123-152; ib., p. 149.

9.Voir par exemple J. Croquison, Les origines de l'iconographie grégorienne, dans : Cahiers Archéologiques. Fin de l'Antiquité et Moyen Âge, 12, 1962, pp. 249-260.

10.Pour ce qui est de la corrélation entre l'incendie de livres/bibliothèques et celui d'hommes dans l'histoire et la littérature, voir les exemples donnés par Leo Löwenthal, Calibans Erbe, dans : Neue Rundschau, 95, 1984, pp. 5-19. En effet, les incinérations de livres ont bien souvent dans l'histoire la fonction d'une liquidation rituelle de l'individu dans sa subjectivité.

11. Pour cet épisode, voir l'éd. Paul Meyer, dans : Romania, 12, 1883, pp. 190-191. La Vie de saint Grégoire le Grand de Angier est la traduction d'une Vita latine rédigée par Jean le Diacre de 873 à 876, traduction qui augmente considérablement l'original (Migne, PL 75, col. 41-242). Le traducteur a mal compris certains passages du texte. Notons la différence entre "Quant a son cors ne porent nuire » et deficiente personali materie : l'autodafé des livres résulte selon le texte latin du défaut de biens personnels du pape, et non pas suite à l'absence du pape lui-même.

12.Wolfgang Speyer, «Büchervernichtung», p. 148.

13.Dans le chapitre «Ceci tuera cela » de Notre-Dame de Paris: «Or quelle immortalité précaire que celle du manuscrit! [...] pour détruire la parole écrite il suffit d'une torche et d'un turc [...] Au quinzième siècle tout change [...] Sous la forme imprimerie, la pensée est plus impérissable que jamais... » (Victor Hugo, CEuvres complètes, Club français du livre, tome XII, pp. 209-210).

14.Wolfgang Speyer, "Die Legende von der Verbrennung der Werke Papst Gregors I », Jahrbuch für Antike und Christentum, 13, 1970, pp. 78-82; ibid., p. 82.

15.D'une manière générale d'ailleurs, Grégoire passe pour avoir été un destructeur fanatique du patrimoine culturel païen ; voir par ex. t. Buddensieg, "Gregory the Great, the Destroyer of Pagan Idols. The History of a Medieval Legend Concerning the Decline of Ancient Art and Literature ", Journal of the Warburg and Courtauld Institutes, 28, 1965, pp. 44-65.

16.W. Speyer, Büchervernichtung und Zensur des Geistes bei Heiden, Juden und Christen, Stuttgart 1981, pp.177-179. Les livres sont considérés comme armes du diable.

17.En effet, cette «histoire de bibliothèque » figure uniquement dans la Vita de Jean le Diacre, la cinquième description de la vie de Grégoire dans l'ordre chronologique. Il est très difficile de juger dans quelle mesure elle correspond à la réalité biographique (voir Jeffrey Richards, Gregor der Große. Sein Leben - seine Zeit, Graz-Wien-Köln, 1983, p. 80). 
18.Hagen Keller, «Vom « heiligen Buch» zur «Buchführung». Lebensfunktionen der Schrift im Mittelalter », Frühmittelalterliche Studien, 26, 1992, pp. 1-31; ibid., p. 5. 19.Vv. 35-38, 61sq. ; éd. Ian Short, Philippe de Thaon. Comput, London, 1984, p. 5. 20.Voir Max Grosse, Das Buch im Roman. Studien zu Buchverweis und Autoritätszitat in altfranzösischen Texten, München 1994. Les exemples cités montrent en effet que peu à peu le romans acquiert lui aussi le statut de livre.

21.Cité d'après l'éd. Paul Meyer, Le bestiaire de Gervaise, dans : Romania, 1, 1872, pp. 410-443. On sait qu'armarium (a. fr. armaire - voir Godefroy VIII, p. 183 et ToblerLommatzsch I, pp. 533-534) est au Moyen Âge le terme usuel pour désigner une bibliothèque dans les deux sens que ce mot a aujourd'hui. Il est alors en concurrence avec livraire/libraire. Ce n'est qu'au $\mathrm{XV}^{\mathrm{e}}$ siècle que bibliothèque prend le dessus. 22.Les Miracles de Notre Dame, éd. V. Frédéric Koenig, tome IV, Genève 1979, p. 134. 23.Tome III, Genève 1966, p. 265. Voir aussi la version de Gautier de l'évangile apocryphe de l'enfance de Jésus : «Á saint Maart ou grant livraire / Trouvai .i. livre en .i. aumaire " (Robert Reinsch, Die Pseudo-Evangelien von Jesu und Maria's Kindheit, Halle 1879, p. 40, vv.1-2).

24.Selon ses propres dires, Willame est le premier prénom d'Adgar (v. 26sq.) : voir le prologue dans : Carl Neuhaus (éd.), Adgar's Marienlegenden nach der Londoner Handschrift, Wiesbaden, 1886, pp. 8-9.

25. «C'est escrit fine, Deu merci! / Selunc le liure Mestre Albri, / Ke de saint Pol oi de l'alamarie. / Plus ne truis en l'essemplarie... » ( ${ }^{\circ} 40$, vv. 528-532).

26.Raymond T. Hill, «La Vie de Sainte Euphrosine », The Romanic Review, 10, 1919, pp. 191-232.

27.Le livre des miracles de Notre-Dame de Chartres, écrit en vers, au XIII ${ }^{\mathrm{e}}$ siècle, par Jehan le Marchant, publié par M. G. Duplessis, Chartres 1855.

28.Monique-Cécile Garand fait remarquer que les bibliothèques monacales intégraient fréquemment leurs livres précieux dans le trésor de l'église (thesauraria) : «Les anciennes bibliothèques du XIII ${ }^{\mathrm{e}}$ au XV $\mathrm{XV}^{\mathrm{e}}$ siècle», dans : A. Vernet (éd.), Histoire des Bibliothèques médiévales, p. 53.

29.Li Romans de Bauduin de Sebourc, III roy de Jhérusalem. Poème du XIVe siècle, publié pour la première fois..., Valenciennes 1841, tome I, p. 123.

30. La Queste del Saint Graal, éd. Albert Pauphilet, Paris, 1967 (CFMA), p. 279-280.

31. "Ceste estoire trovons escrite [...] An un des livres de l'aumeire / Mon seignor saint Pere a Biauvez. / De la fu li conte estrez... » (vv. 18-22; Kristian von Troyes, Cligés, éd. Wendelin Foerster, Halle a. d. S. 1921, p. 1).

32.Localisation qui se retrouve dans le prologue de la chanson de geste Orson de Beauvais (v. 2528sq. de l'éd. Gaston Paris, Paris, 1899 [SATF]).

33. Voir à ce sujet Friedrich Ohly, «Vom geistigen Sinn des Wortes im Mittelalter », article repris dans : id., Schriften zur mittelalterlichen Bedeutungsforschung, Darmstadt 1977, pp. 1-31.

34.Citations d'après l'éd. L. Constans, Paris, 1904-1912 (SATF).

35.Version du ms. Paris, Bibliothèque nationale, fonds français 1627 ; voir l'épilogue en trois versions chez H. F. Williams, dans : Romance Philology, 7, 1953/54, pp. 143-155.

36. Même le verbe inventer formé au milieu du $\mathrm{XV}^{\mathrm{e}}$ siècle et remplaçant trouver pour désigner l'acte poétique, signifie également encore simplement «trouver»au début. Encore chez Christine de Pizan, dans le Livre de la Cité des Dames, à la scène de 
l'inspiration, qui témoigne d'une haute conception de soi en tant que poétesse et femme, et à l'analogie patente avec la scène biblique de l'Immaculée Conception de ("...soubdainement sus mon giron vy descendre un ray de lumiere...»), sont accentués la passivité et le caractère non autonome du «trouver » poétique, analogues au «trouver par hasard dans une bibliothèque " (voir ce passage dans l'éd. M. Ch. Curnow, thèse Vanderbilt University 1975, pp. 621-622).

37.La Couleur de la mélancolie. La fréquentation des livres au XIVe siècle. 1300-1415, Paris, 1993, p. 128.

38.Bonaventure Des Périers, Cymbalum mundi, éd. P.H. Nurse, Manchester-London 1957, p. 3.

39. Euvres poétiques de Guillaume Crétin, éd. K. Chesney, Paris, 1932, p. 174 (vv. 541-545).

40. Reproduite chez J. Cerquiglini-Toulet, La Couleur de la mélancholie.

41.François Villon, Cuvres, éd. Auguste Longnon, Paris, 1967 (Classiques français du Moyen Âge), Le lais, v. 284sq.

42.En ce qui concerne le classement effectif des livres dans les divers types de bibliothèques médiévales, voir en détail Donatella Nebbiai-Dalla Guardia, "Classifications et classements ", dans: A. Vernet (éd.), Histoire des bibliothèques françaises. Les bibliothèques médiévales, pp. 373-393. La grande diversité de ces systèmes de classement et d'archivage, répondant généralement à des critères pragmatiques, ne contredit pas une organisation fondamentalement centrée sur la Bible ou du moins les libri divini ou libri canonici.

43.Voir Horst Wenzel, Hören und Sehen. Schrift und Bild. Kultur und Gedächtnis im Mittelalter, München 1995, p. 351sq.

44.Aleida Assmann, Erinnerungsräume. Formen und Wandlungen des kulturellen Gedächtnisses, München 1999, p. 115.

45.Gerd Schmidt, «Waffenlärm und Grabesstille. Buch und Bibliothek im Spiegel der Metapher ", Philobiblon, 34, 1990, pp. 3-12; ib., p. 4. Cette sentence (Migne, PL 205, col. 845) a été maintes fois répétée et déformée jusqu'à Umberto Eco (Monasterium sine libris est sicut civitas sine opibus, castrum sine numeris... ; Il nome della rosa, Milano 1980, p. 44).

46.Ernst Robert Curtius, Europäische Literatur und lateinisches Mittelalter, Bern $1961^{3}$, p. 324 .

47.Migne, PL 101, col 745 ( $n^{\circ}$ LXVII).

48.Jan-Dirk Müller, «Das Gedächtnis der Universalbibliothek : Die neuen Medien und der Buchdruck", dans : Hartmut Böhme-Klaus R. Scherpe (éd.), Literatur- und Kulturwissenschaft, Hamburg, 1996, pp. 78-95; ib., p. 81.

49.Voir à ce sujet Alastair J. Minnis, Medieval Theory of Authorship. Scholastic Literary Attitudes in the later Middle Ages, London, 1984, pp. 101-102.

50.Voir Bernard Ribémont, De Natura Rerum. Études sur les encyclopédies médiévales, Orléans 1995 (Medievalia 18), ainsi que son introduction dans : Le livre des propriétés des choses. Une encyclopédie au XIVe siècle, Paris, 1999, pp. 7-49.

51.Voir les exemples donnés par M. Grosse, Das Buch im Roman, p. 90sq.

52.Le fait que peu à peu, du moins partiellement au sein de certains chapitres et parties, l'ordre alphabétique s'impose (surtout d'abord là où un classement systématique se révèle problématique), est à voir dans ce contexte ; voir B. Ribémont, dans : Le Livre des propriétés..., pp. 27-28. Le pragmatisme, axé sur l'utilisateur, de l'ordre alphabétique dans les encyclopédies médiévales, lesquelles ont essentiellement une 
vocation éducative, n'a toutefois pas grand chose à voir avec le classement alphabétique des encyclopédies modernes, qui, lui, est l'expression de la décentralisation et de la complexité croissante du savoir, devenue impossible à systématiser.

53.J. Cerquiglini-Toulet, La Couleur de la mélancolie, p. 58.

54.Voir René d'Anjou, Le Livre du cuer d'Amours espris, éd. S. Wharton, Paris, 1980, p. 141. 55.Voir la liste qu'en dresse W. Speyer dans Büchervernichtung und Zensur, p. 120sq.

56.Illustrations $i b$., pp. 185-187.

57.Voir le catalogue de l'exposition Glaube und Wissen im Mittelalter. Die Kölner Dombibliothek (Erzbischöfliches Erzdiözesanmuseum, Cologne, 7 août-15 nov. 1998, München 1998, p. 14 et les remarques p. 15, 187-190.

58.Citations d'après l'édition Riccardo da Bury, Philobiblon, edizione critica a cura di Antonio Altamura, Napoli 1954.

59.Voir au chapitre VII le regret exprimé au sujet de la destruction en Égypte des livres des Ptoléméens au cours de la première guerre d'Alexandrie, c'est-à-dire concernant l'incendie déjà mythique au Moyen Âge de la bibliothèque de la ville. On y trouve aussi ce qui constitue (probablement) le premier catalogue d'une bibliothèque disparue dans la littérature mondiale.

60.Au chapitre IX, Richard de Bury prolonge la ligne (Proche-Orient)-Athènes-RomeParis, en ajoutant l'Angleterre, «la plus célèbre de toutes les îles » («insularum insignissima ", 108).

61.Il s'agit là des vers 3 et 4 du prologue de l'Ars Phocae (Grammatici latini, éd. H. Keil, tome V, Leipzig 1868, p. 410).

62. Voir Robert Guiette, D'une poésie formelle en France au Moyen Âge (1949), dernièrement dans : id., «Questions de littérature », Romanica Gandensia, 8, 1960, pp. 9-23.

63. Édition: La Biblionomia de Richard de Fournival, texte en facsimilé avec la transcription de Léopold Delisle, Pretoria 1965 (Mousaion 62).

64.Voir l'édition de Charles Henry Beeson, Isidor-Studien, München, 1913, (Quellen und Untersuchungen zur lateinischen Philologie des Mittelalters IV, 2), pp. 157-163.

65.Voir Jan Bialestocki, «Bücher der Weisheit und Bücher der Vergänglichkeit. Zur Symbolik des Buches in der Kunst ", Abhandlungen der Heidelberger Akademie der Wissenschaften. Philosophisch-historische Klasse, Jahrgang, 1984, 5 Abhandl., Heidelberg, 1984, p.3sq.

66.Ce qui suit s'appuie en partie sur les indications de Fritz Nies, «Monde des livres Livre du monde : Le poète-lecteur et sa représentation iconographique », dans : JeanClaude Ternaux (éd.), La Naissance du monde et l'invention du poème. Mélanges de poétique et d'histoire littéraire du XVI ${ }^{e}$ siècle offerts à Yvonne Bellenger, Paris, 1998 (Études et essais sur la Renaissance 21), p. 37-54; ibid., p. 40sq.

67.Etagère tournante, souvent à deux niveaux, que l'on rencontre en particulier dans l'iconographie de saint Jérôme.

68.F. Nies, Monde des livres, p. 42.

69.Reproduction dans : Franco Cardini, M.T. Fumagalli-Beonio-Brocchieri, Universitäten des Mittelalters. Die europäischen Stätten des Wissens, München, 1991, p. 200.

70.Le lien entre isolation et domination est repris dans ce qui suit : «C'est là mon siège. J'essaie à m'en rendre la domination pure, et à soustraire ce seul coin à la communauté et conjugale, et filiale, et civile... ». 
71.Klaus Schreiner informe en détail, maints documents et exemples à l'appui, des résistances sociales et religieuses : «Laienbildung als Herausforderung für Kirche und Gesellschaft. Religiöse Vorbehalte und soziale Widerstände gegen die Verbreitung von Wissen im späten Mittelalter und in der Reformation », Zeitschrift für historische Forschung, 11, 1984, pp. 257-354.

\section{AUTEUR}

DIETMAR RIEGER

Université de Giessen 